\title{
Effect of the beta secretase-1 inhibitor on the amyloid C-terminal fragment of amyloid precursor protein processing in a hyperphosphorylated tau rat model
}

\author{
H.Y. Chen ${ }^{1}$, L. Wang ${ }^{2}$, J.F. Liu ${ }^{3}$, W.Z. Wang ${ }^{1}$ and C.J. Yu ${ }^{1}$ \\ ${ }^{1}$ Department of Neurology, \\ The Second Affiliated Hospital of Harbin Medical University, \\ Harbin, Heilongjiang, China \\ ${ }^{2}$ Department of Geriatrics, \\ The Second Affiliated Hospital of Harbin Medical University, \\ Harbin, Heilongjiang, China \\ ${ }^{3}$ Department of Neurology, The First Hospital of Harbin, \\ Harbin City, Heilongjiang, China \\ Corresponding authors: W.Z. Wang / C.J. Yu \\ E-mail: cnhydoc@126.com \\ Genet. Mol. Res. 13 (3): 6213-6227 (2014) \\ Received June 10, 2013 \\ Accepted February 17, 2014 \\ Published August 15, 2014 \\ DOI http://dx.doi.org/10.4238/2014.August.15.4
}

\begin{abstract}
The amyloid C-terminal fragment ( $\beta C T F)$ of the amyloid precursor protein (APP) is the cleaved component of APP by beta secretase-1 (BACE1), which shows similar neurotoxicity as amyloid beta $(\mathrm{A} \beta)$ in many ways. Evidence suggested that in addition to $\mathrm{A} \beta, \beta \mathrm{CTF}$ might also participate in the pathogenesis of Alzheimer's disease (AD). In recent years, the relationship between $\beta C T F$ processing and hyperphosphorylated tau has attracted increasing research attention. In this study, we established an animal model of tau hyperphosphorylation with okadaic acid (OA) treatment, and analyzed $\beta C T F$ processing in vivo. The $\beta C$ TF level was found to increase in
\end{abstract}


neurons, which was most likely caused by the induction of OA and BACE1 overexpression. Furthermore, these results provide the first evidence that $\beta \mathrm{CTF}$ can predominately accumulate in the axons of neurons in a hyperphosphorylated tau state in vivo, and suggested that the redistribution of $\beta C T F$ is involved in the pathogenesis of $A D$. These results indicate that $\mathrm{BACE} 1$ could be a therapeutic target of $\mathrm{AD}$ by affecting the processing of $\beta C \mathrm{TF}$.

Key words: Alzheimer's disease; Amyloid C-terminal fragment; BACE1; Tau hyperphosphorylation

\section{INTRODUCTION}

Alzheimer's disease (AD) is an age-related neurodegenerative disorder, which is recognized as the most common form of dementia, and seriously affects quality of life causing great psychological and economic burdens to patients and their families. The disease is pathologically characterized by neuronal degeneration, neuritic plaques containing amyloid beta $(\mathrm{A} \beta)$, and neurofibrillary tangles containing hyperphosphorylated tau. The $\mathrm{A} \beta$ peptide and the tau protein are not only pathological features of $\mathrm{AD}$, but are also involved in the pathogenesis of AD (Gong and Iqbal, 2008).

To date, the cause and progression of $\mathrm{AD}$ have not been fully elucidated. There are many hypotheses about the pathogenesis of $\mathrm{AD}$. Within the last decade, the amyloid cascade hypothesis was proposed to expound the main cause of $\mathrm{AD}$, in which the oligomerization and accumulation of $\mathrm{A} \beta$ was thought to be the primary cause of $\mathrm{AD}$ and the main cause of other disease processes (Hardy, 2006). However, there is some controversy related to this hypothesis. A large amount of $A \beta$ was found to be generated in cell lines of tau hyperphosphorylation, which could not be clearly explained by the amyloid cascade hypothesis (Fukumoto et al., 2002). In addition, another report showed that in mice overexpressing wild-type human amyloid precursor protein (APP), memory deficits and increasing levels of phosphorylated tau occurred early, whereas $A \beta$ was not detected, which supported the view of an $A \beta$-independent pathogenic pathway in AD (Simón et al., 2009). Other studies have indicated that tau phosphorylation plays an important role in AD pathology (Oddo et al., 2006; Roberson et al., 2007). Moreover, several lines of evidence have shown that $\mathrm{A} \beta$ might not be the only component involved in the pathogenesis of $\mathrm{AD}$, and that other cleavage fragments of APP, such as the amyloid C-terminal fragment $(\beta \mathrm{CTF})$, might participate in the pathophysiology of AD (Chang and Suh, 2005). The $\beta C T F$ shows much higher neurotoxicity than $A \beta$, including neurodegeneration, endosome dysfunction, and synaptic or memory deficits (Choi et al., 2001; Lee et al., 2006).

Exploration of the expression and distribution of $\beta$ CTF might help to reveal its biological activity and to better understand its involvement in neurodegeneration, which could pave the way for developing a novel therapeutic approach for AD (Lahiri et al., 2002). The relationship between $\beta C T F$ processing and tau phosphorylation has attracted much attention recently; however, few studies have examined whether tau phosphorylation has an effect on $\beta C T F$ processing or the possible underlying mechanism (Yoon et al., 2006). In our previous studies, we established a hyperphosphorylation tau cell model by treating primary rat neurons with the protein phosphatase inhibitor okadaic acid (OA), and found that the level of neurotoxic 
$\beta C T F$ increased, indicating that hyperphosphorylated tau might have an effect on the $\beta C T F$ processing in vitro (Yu et al., 2008). However, the effect that hyperphosphorylated tau might have on $\beta \mathrm{CTF}$ in vivo is not clear, which might provide further insight into the pathogenesis of $\mathrm{AD}$, and help in the search for appropriate therapeutic targets.

Herein, we developed an experimental tau hyperphosphorylation animal model via OA microinfusion. We examined the expression of $\beta C T F$ in vivo and found that $\beta C T F$ increased, which was mainly due to the overexpression of beta secretase-1 (BACE1) under the hyperphosphorylated tau state. Importantly, we first found that the distributions of $\beta C T F$ changed in the hyperphosphorylated tau state. Moreover, as a potential therapeutic approach for treating $\mathrm{AD}$, the effect of $\mathrm{BACE} 1$ inhibitor on $\beta \mathrm{CTF}$ processing was also investigated in the OA-induced tau hyperphosphorylation rat model.

\section{MATERIAL AND METHODS}

\section{Experimental animals}

The experiments were carried out with adult male Sprague-Dawley rats (250-320 g). All rats were housed in a room with a $12 / 12$-h light/dark cycle at $22^{\circ} \pm 2{ }^{\circ} \mathrm{C}$ with free access to water and food. The experimental protocol and procedures were conducted in accordance with the regulations of the Ethics Committee of Harbin Medical University.

\section{Animal groups and drugs}

Rats were randomly divided into the following four groups with 6 rats in each group. Group I (control group) received no treatment. Group II (sham group) received $1 \mu \mathrm{L} 100 \%$ ethanol by intracerebralventricular (icv) bilateral injection, which was applied on days 1 and 3 , and $2 \mu \mathrm{L} 100 \%$ ethanol was administered on day 21. In group III (OA group), OA was dissolved in $100 \%$ ethanol and $1 \mu \mathrm{L} 0.4 \mathrm{mM} \mathrm{OA}$ was icv injected bilaterally on days 1 and 3 , and $2 \mu \mathrm{L} 100 \%$ ethanol was administered on day 21. In group iv (OA + BACE1 inhibitor group), OA was dissolved in $100 \%$ ethanol and $1 \mu \mathrm{L} 0.4 \mathrm{mM}$ OA was $i c v$ bilaterally injected on days 1 and 3; BACE inhibitor $i v$ (10 mg BACE inhibitor $i v$ in $2 \mu \mathrm{L} \mathrm{100 \%} \mathrm{ethanol)} \mathrm{was} \mathrm{administered}$ on day 21.

Once the models were established, testing was conducted according to the plan shown in Figure 1.

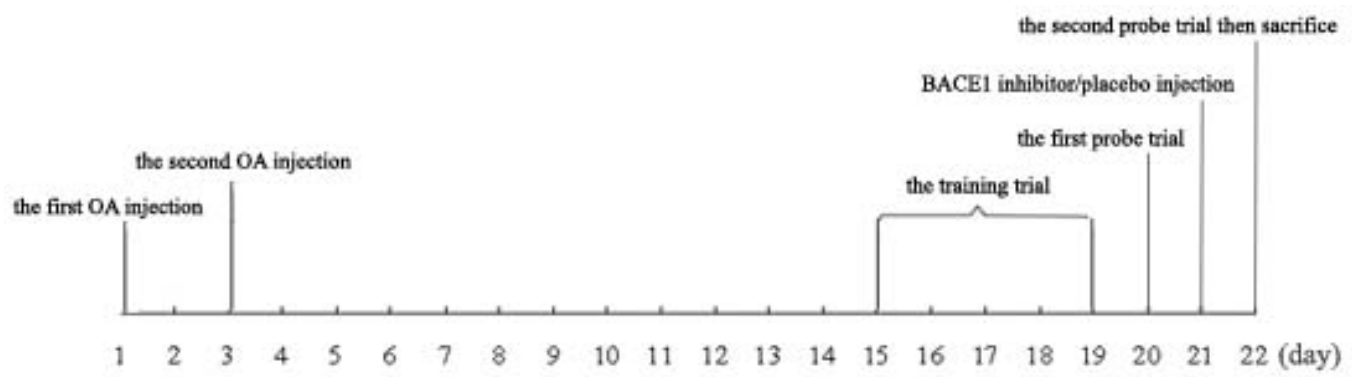

Figure 1. Testing schedule. 


\section{Animal model and treatments}

On the day of surgery, rats were intraperitoneally administered general anesthesia (300 $\mathrm{mg} / \mathrm{kg}$ chloralhydrate), their heads were positioned on a stereotaxic apparatus, and a midline sagittal incision was made in the scalp. Once the skull was exposed, two holes were drilled 1.0 $\mathrm{mm}$ posterior to the bregma, $1.5 \mathrm{~mm}$ lateral to the sagittal suture, and $5.0 \mathrm{~mm}$ beneath the surface of the brain. OA, BACE inhibitor $i v$, or placebo was then injected with a microsyringe. After injection, the microsyringe was held for $10 \mathrm{~min}$ to allow sufficient dispersion of the solution. After the operation, rats were placed on a heating pad to maintain body temperature and were kept there until they recovered from the anesthesia. All operations were performed under sterilization.

\section{Sacrifice and tissue preparation}

The second behavior rat test was employed. Rats were anesthetized and transcardially perfused with approximately $200 \mathrm{~mL}$ normal saline. The brain was carefully removed from the skull, and the hemispheres used for immunohistochemistry were fixed in $4 \%$ paraformaldehyde for $48 \mathrm{~h}$ at $4^{\circ} \mathrm{C}$, and then embedded in paraffin after being coronally sliced into 3-mm sections. The hippocampus tissues used for Western blotting analysis and real-time reverse transcriptase-polymerase chain reaction (RT-PCR) were immediately frozen in liquid nitrogen and stored at $-80^{\circ} \mathrm{C}$.

\section{Western blotting}

The hippocampuses were homogenized in cold lysis buffer. The homogenates were then sonicated for $2 \mathrm{~min}$, centrifuged at $12,000 \mathrm{~g}$ for $30 \mathrm{~min}$ at $4^{\circ} \mathrm{C}$, and the supernatants were collected for protein concentration measurement and the Western blotting assay. Proteins of the hippocampus were separated by sodium dodecyl sulfate-polyacrylamide gel electrophoresis and the separated protein bands were transferred to polyvinylidene difluoride membranes, which were subsequently blocked with 5\% skimmed milk in Tris-buffered saline for $1 \mathrm{~h}$. Membranes were incubated overnight at $4^{\circ} \mathrm{C}$ with appropriate primary antibodies, respectively, including anti-phospho tau AT8 (Santa Cruz Biotechnology, Santa Cruz, CA, USA), rabbit anti- $\beta$-APP (Invitrogen), and mouse anti-BACE monoclonal antibody (Santa Cruz Biotechnology), and the concentrations were chosen according to results of preliminary experiments. The appropriate secondary antibodies were then applied. The membranes were washed for 5 min after blocking and incubation steps were performed. The membranes were developed using an ECL Western blotting system and were quantitated by Kodak image analysis. All blots were normalized by $\beta$-actin.

\section{Immunohistochemistry}

Paraffin-embedded brains were coronally sliced into 5-mm-thick sections on a vibratome. The sections were dewaxed and washed in distilled water, immersed in citrate buffer solution in order to recover the antigen, sequentially bathed in $3 \% \mathrm{H}_{2} \mathrm{O}_{2}$, blocked in $10 \%$ goat serum, and incubated overnight at $4{ }^{\circ} \mathrm{C}$ with the primary antibodies: anti-phospho tau AT8 or rabbit anti- $\beta$-APP polyclonal antibody (APP-CT; Invitrogen). The sections were then treated with appropriate secondary antibodies. Sections were all washed with phosphate-buffered sa- 
line 3 times for 5 min after each step. To count the positive immunoreactive cells in the hippocampus of the rats, three sections per rat were selected, and 5 comparable fields were selected randomly.

\section{Behavioral water maze test}

A circular pool, $120 \mathrm{~cm}$ in diameter and $50 \mathrm{~cm}$ high, was used as the water maze. Black waterproof paper was stuck to the inner side of the pool so that rats could not see the platform when they were swimming. The pool was filled with water, and the temperature of the water was maintained at $24.0^{\circ} \pm 0.5^{\circ} \mathrm{C}$. The pool was divided into four quadrants, and the invisible transparent platform located $1.0 \mathrm{~cm}$ below the water surface was placed in the center of the third quadrant. During the training trial, a rat was placed on the platform for about $30 \mathrm{~s}$, and then started from the other three quadrants without platforms, and the trial ended once it climbed the platform. A maximum time of $120 \mathrm{~s}$ was recorded if the rat did not find the hidden platform, and then the rat was allowed to rest on the platform for $30 \mathrm{~s}$. Rats were trained for 5 successive days. The escape latency time (the amount of time the rat spent swimming to find the hidden platform) reflected the rat's spatial learning ability. After training for 5 days, rats were given a probe trial of $90 \mathrm{~s}$ with the platform removed. All rats started in the first quadrant. The time spent in the third quadrant and the number of times crossing the target platform were recorded to reflect spatial memory ability. One day after the BACE1 inhibitor or placebo was administered, the probe trial was performed again.

\section{Quantitative RT-PCR}

Total RNA from the hippocampus was reverse-transcribed into cDNA according to manufacturer instructions. Total RNA was converted to cDNA by reverse transcriptase using a SuperScript ${ }^{\mathrm{TM}}$ II Reverse Transcriptase kit (Invitrogen). The primer sequences used in this study were: APP forward, 5'-GCGGACACAGACTATGCTGA-3' and reverse, 5'-CTCTGTG GCCTCTTCGTAGG-3'; BACE1 forward, 5'-GCTGCAGTCAAGTCCATCAA-3' and reverse, 5'-ATTGCTGAGGAAGGATGGTG-3'; $\beta$-actin forward, 5'-CACTTTCTACAATGAG CTGCG-3' and reverse, 5'-CTGGATGGCTACGTACATGG-3'.

\section{Statistical analysis}

Differences among groups were compared by one-way analysis of variance (ANOVA) followed by the Student-Newman-Keuls test if there was a significant difference between groups. Values are reported as means $\pm \mathrm{SE}$, and $\mathrm{P}<0.05$ was considered to be statistically significant for all experiments.

\section{RESULTS}

\section{OA icv injection induced tau hyperphosphorylation}

Results of Western blot analysis showed that phospho-tau levels of the hippocampus were significantly higher in the OA group compared to the control group (Figure 2A). Positive 
neurons for AT8 staining were found in the OA group and in the OA + BACE1 inhibitor group, but were not present in sections from the control group and the sham group (Figure 2B).

A

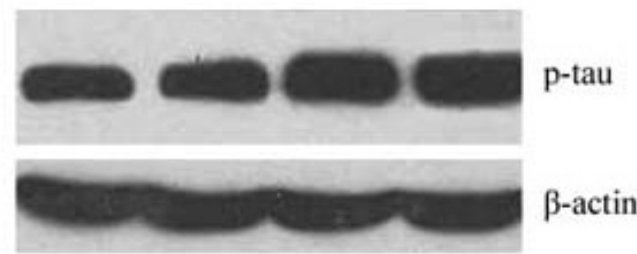

$\square$ control group

- sham group

$\square$ OA group

$\square \mathrm{OA}+\mathrm{BACE} 1$ inhibitor group

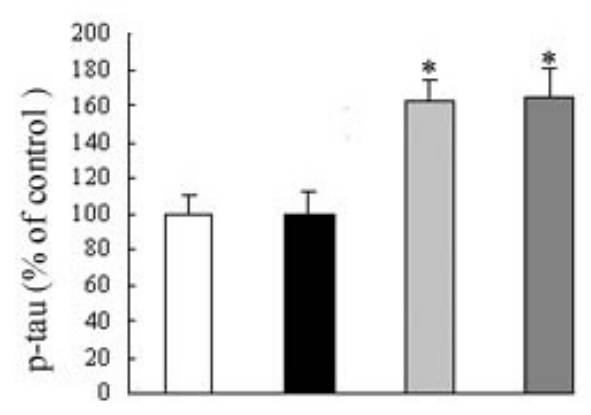

B

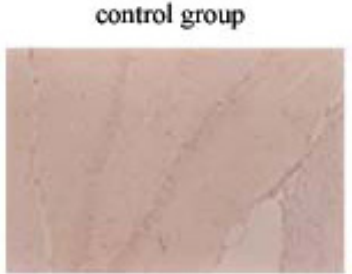

sham group

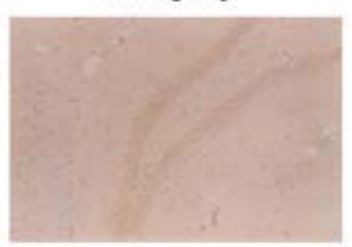

OA group

$\mathrm{OA}+\mathrm{BACE} 1$ inhibitor group
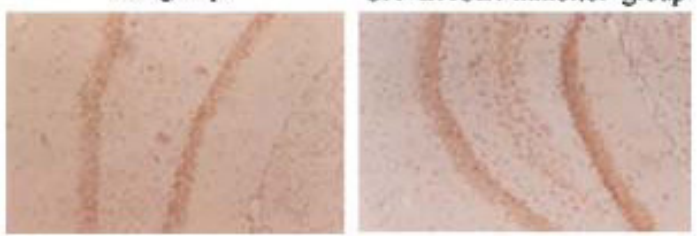

Figure 2. Tau phosphorylation increased in rats given okadaic acid (OA). A. Western blot analysis showed that tau was hyperphosphorylated in the hippocampus, ${ }^{*} \mathrm{P}<0.05$. B. Hyperphosphorylated tau was observed in some of the hippocampal neurons of rats given OA. 


\section{Expression of $\beta C T F$ increased}

APP was proteolyzed by BACE1 to produce $\beta \mathrm{CTF}$ (C99 and C89) and by $\alpha$-secretase to produce $\alpha \mathrm{CTF}$ (C83). The level of $\beta C T F$ in the OA group was increased significantly compared with that of the control group $(\mathrm{P}<0.05)$, and a significant reduction was observed in the $\mathrm{OA}+$ BACE1 inhibitor group compared to the OA group $(\mathrm{P}<0.05)$ (Figure 3). However, the $\mathrm{C} 83$ level showed no apparent changes among the four groups (data not shown). There was no significant difference in the $\beta C T F$ levels between the control group and the sham group $(\mathrm{P}>0.05)$. These results suggested that APP was mainly processed by $\beta$-secretase rather than by $\alpha$-secretase, and that the path of APP processing changed under the tau hyperphosphorylation state.
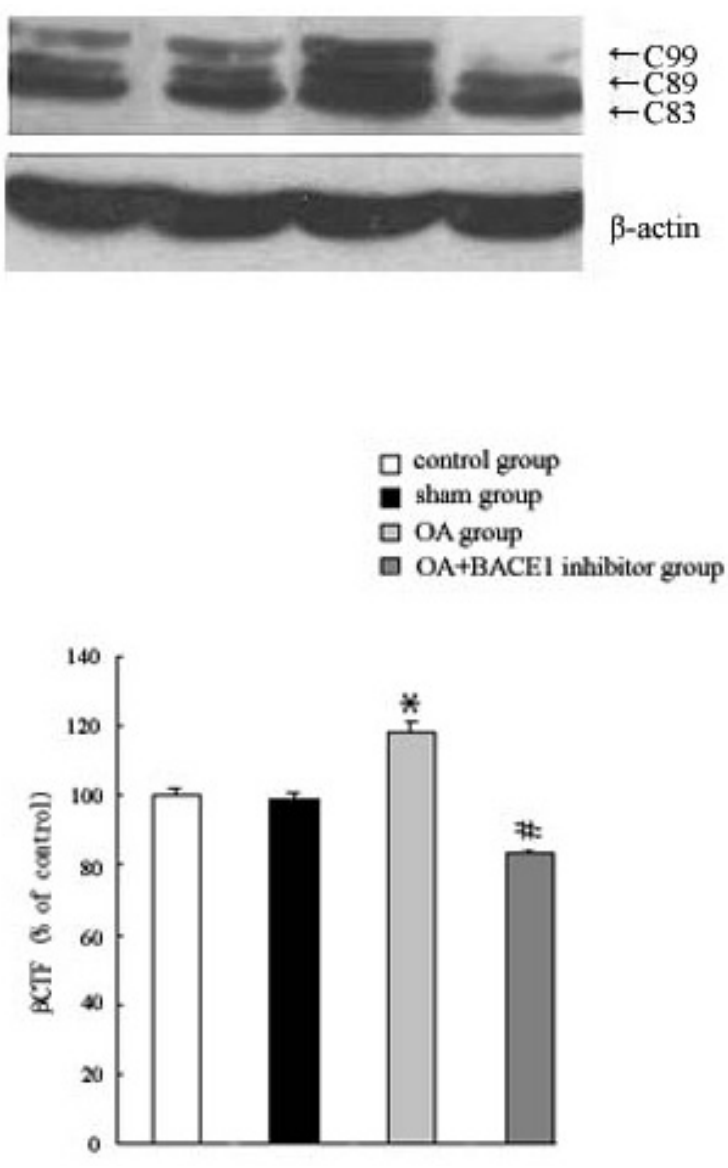

Figure 3. $\beta C T F$ in the hippocampus of the four groups. ${ }^{*} \mathrm{P}<0.05$ compared with the control group and ${ }^{*} \mathrm{P}<0.05$ compared with the okadaic acid $(\mathrm{OA})$ group.

\section{Expression of full APP had no significant change}

To determine the cause of the increase in $\beta C T F$, the level of full APP was measured by Western blot analysis and qRT-PCR. The results showed that the protein level of full APP in 
the OA + BACE1 inhibitor group was slightly increased compared with that of the OA group; however, the difference was not statistically significant $(\mathrm{P}>0.05)$ (Figure 4A). The mRNA levels of full APP did not differ significantly among the four groups $(\mathrm{P}>0.05)$ (Figure 4B). These results showed that: i) the OA $i c v$ injection did not change the expression of full APP, ii) the increase in $\beta \mathrm{CTF}$ was most likely not related to the overexpression of full APP, and iii) inhibiting BACE1 led to a slight increase in full APP, although the change was not significant.

A
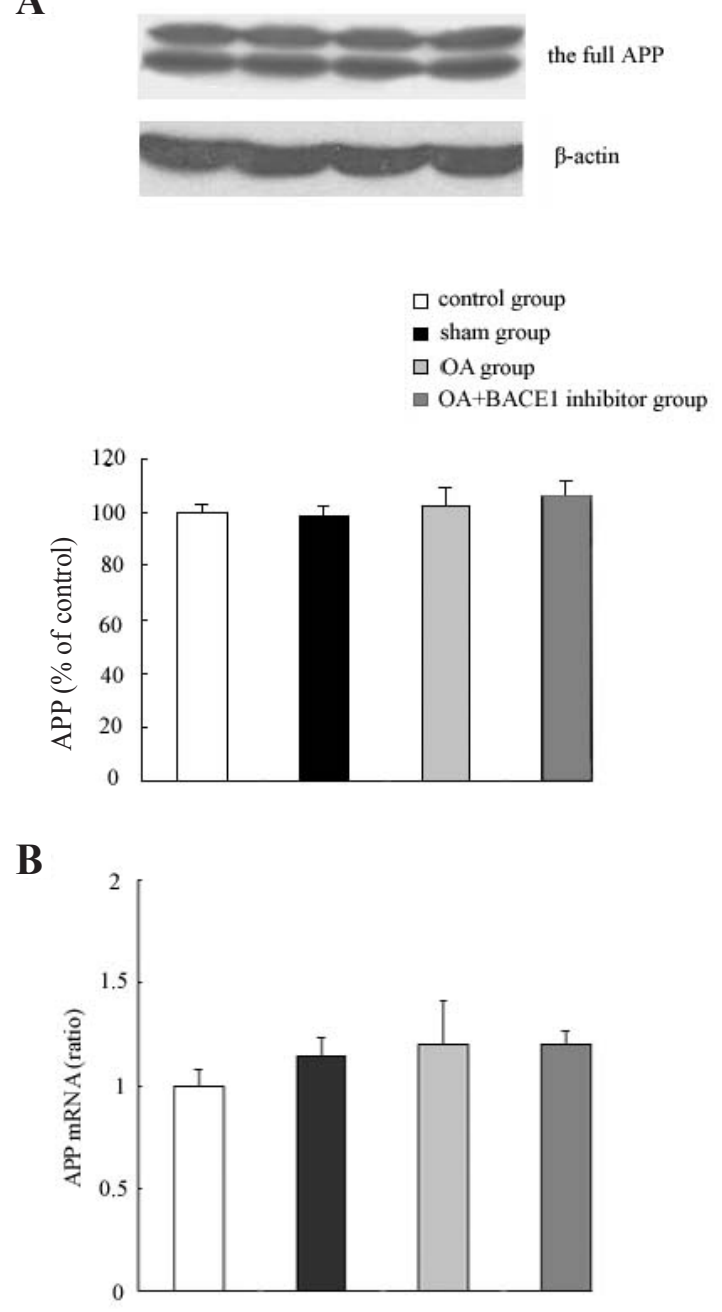

Figure 4. Expression of amyloid precursor protein (APP) in the level of protein (A) and mRNA(B). There were no significant differences in the two levels of APP in the four groups. OA= okadaic acid.

\section{Expression of BACE1 increased}

In addition to investigating the principal precursor of $\beta C T F$, we also detected the expression of BACE1 and found that the protein and mRNA BACE1 levels were significantly 
higher in the OA group compared to the control group $(\mathrm{P}<0.05)$ (Figure 5). Furthermore, the protein expression of BACE1 in the OA + BACE1 inhibitor group was lower than that of the OA group $(\mathrm{P}<0.05)$ (Figure 5A), whereas there was no statistical difference in the level of mRNA between the two groups (Figure 5B). These results suggested that tau hyperphosphorylation contributed to the BACE1 protein and mRNA expression levels, and that the BACE1 inhibitor affected the protein levels but not the mRNA levels. There was no significant difference in the expression of BACE1 between the control group and the sham group $(\mathrm{P}>0.05)$, in spite of the influence of the operation.

Altogether, our data indicated that neurotoxic $\beta C T F$ increased in the hyperphosphorylated tau state, and that the overexpression of BACE1 might be the main cause of this increase. Moreover, BACE1 inhibitor could decrease neurotoxic $\beta$ CTF levels by inhibiting the activity of BACE1 at the protein level.

A
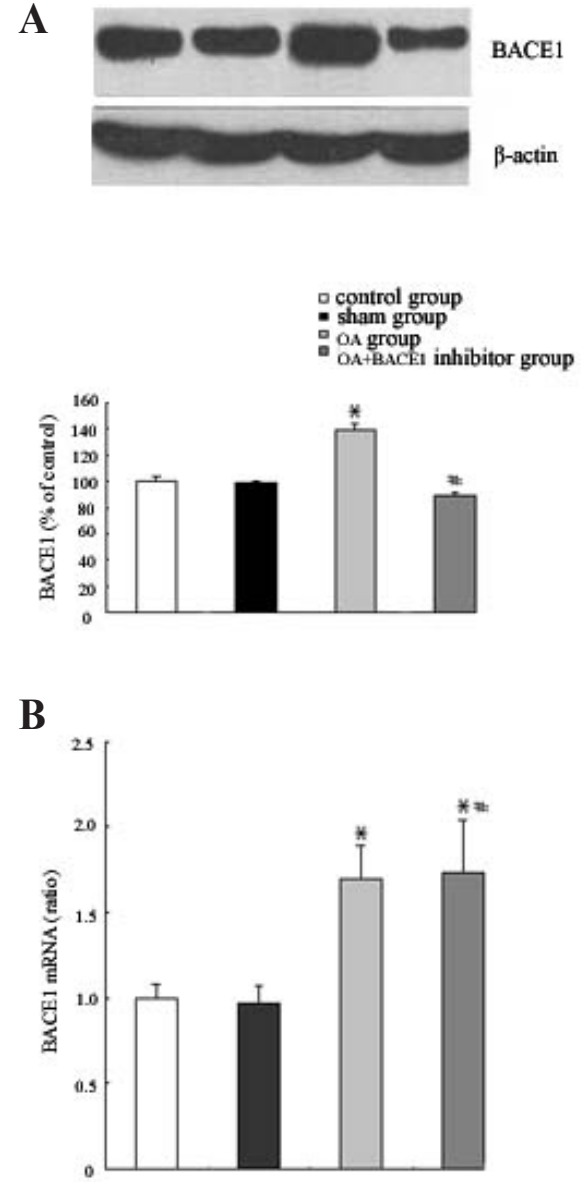

Figure 5. Expression of BACE1 in the level of protein and mRNA. A. Protein level of BACE1 in the hippocampus of the four groups of rats. ${ }^{*} \mathrm{P}<0.05$ compared with the control group and ${ }^{*} \mathrm{P}<0.05$ compared with the okadaic acid (OA) group. B. BACE1 mRNA level increased in the OA group and in the OA + BACE1 inhibitor group. $* \mathrm{P}<0.05$ compared with the control group. However, there was no significant difference between the OA group and the OA + BACE1 inhibitor group $\left({ }^{*} \mathrm{P}>0.05\right)$. 


\section{Distribution of $\beta C T F$ in the hyperphosphorylated tau state}

The APP-CT antibody recognized full-length APP or the membrane-bound APP fragments (CTFs) after cleavage at the $\alpha$-site or $\beta$-site. Use of the APP-CT antibody resulted in granular cytoplasmic immunostaining in the perinuclear region and axonal compartments, and BACE1 inhibitor treatment decreased the immunostaining in the axonal compartments while there was no apparent change in the perinuclear region compartments (Figure 6). This suggested that $\beta \mathrm{CTF}$ had principally accumulated in axons, and BACE1 inhibitor could decrease its deposition in axons.
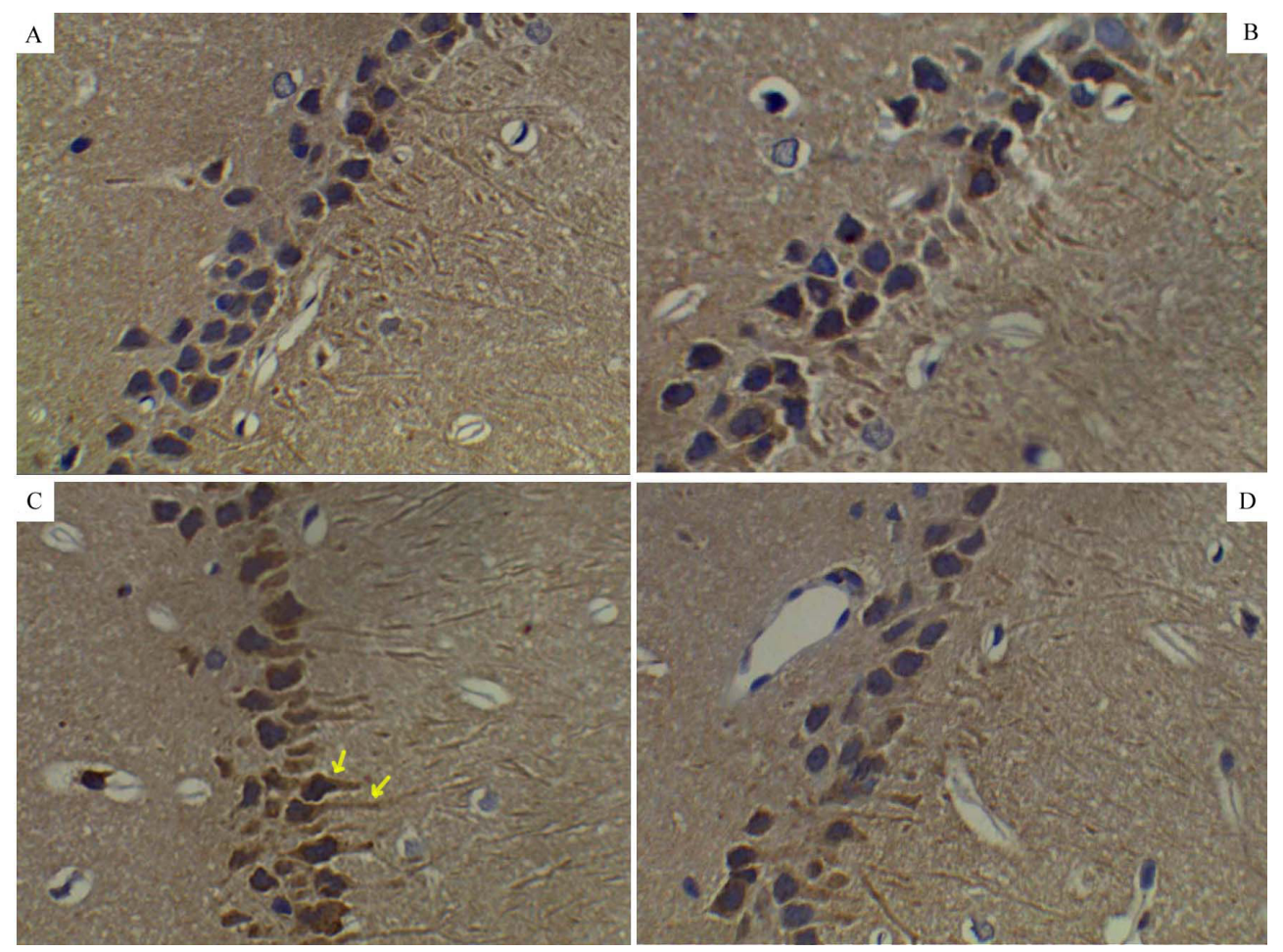

Figure 6. Immunohistochemical analysis for the APP-CT antibody in paraffin sections from the hippocampuses. A. Control group, B. sham group, C. OA group: immunoreactivity grains were mainly distributed in axons (arrows), D. OA + BACE1 inhibitor group: immunoreactivity grains distributed in the axon decreased with BACE1 inhibitor treatment.

\section{Effect of BACE1 inhibitor on memory defects}

During the training trial, the latency time gradually reduced from training of day 3 to day 5 in rats given $\mathrm{OA}$ (Figure 7A), which suggested that $\mathrm{OA}$ induction decreased the spatial learning ability of these rats. The probe trial was first performed on day 20 to evaluate the effect of tau hyperphosphorylation on memory defects. We found that the time remaining in the third quadrant and the number of times the platform was crossed significantly decreased 
after OA treatment (Figure 7B and C). To evaluate the effect of BACE1 inhibitor on memory, the probe trial was conducted once more on day 22. There was a significant change in the time remaining in the third quadrant and in the number of times crossing the platform in the $\mathrm{OA}+$ BACE1 group, whereas there were no significant changes in the control, sham, and OA groups, which were all given placebos (Figure 7D and E). This indicated that OA bilateral icv injection resulted in memory defects, and that BACE1 inhibitor treatment could improve memory ability.

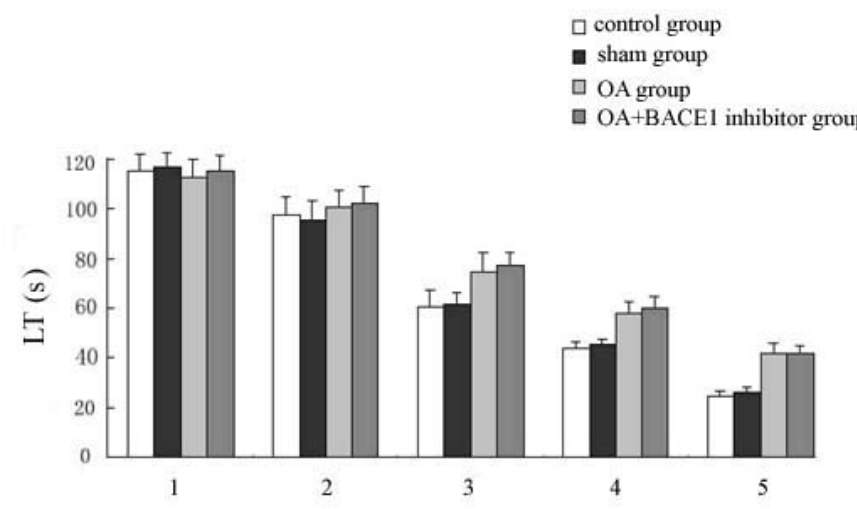

A

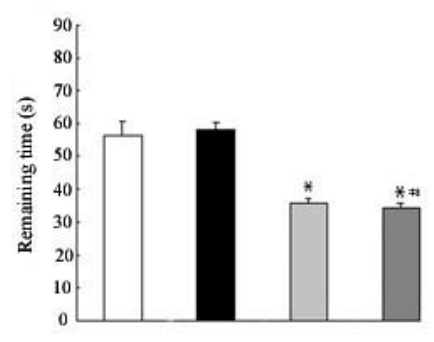

B

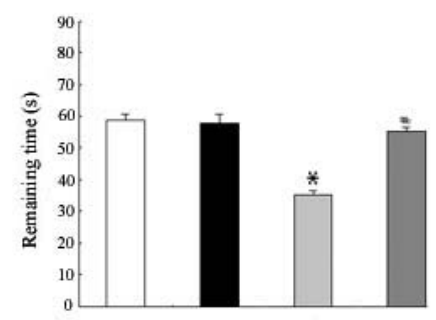

D

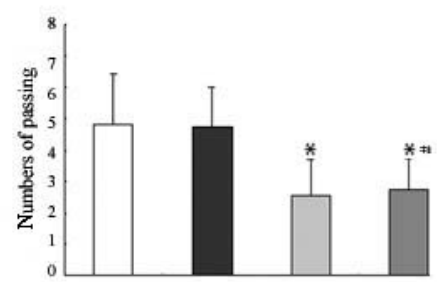

C

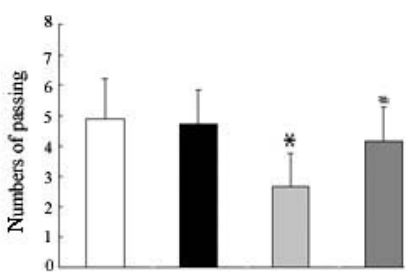

E

Figure 7. Behavioral test with water maze test. A. Training trial. B. Time remaining in the third quadrant in the fist probe trial; $* \mathrm{P}<0.05$ compared with the control group, ${ }^{*} \mathrm{P}>0.05$ compared with the okadaic acid (OA) group. $\mathbf{C}$. Number of times crossing the platform in the fist probe trial; ${ }^{*} \mathrm{P}<0.05$ compared with the control group, ${ }^{\#} \mathrm{P}>0.05$ compared with the OA group. D. Time remaining in the third quadrant in the second probe trial; $* \mathrm{P}<0.05$ compared with the control group, ${ }^{*} \mathrm{P}<0.05$ compared with the OA group. E. Number of times crossing the platform in the second probe trial; ${ }^{*} \mathrm{P}<0.05$ compared with the control group, ${ }^{*} \mathrm{P}<0.05$ compared with the OA group. 


\section{DISCUSSION}

In recent years, drugs such as $N$-methyl-D-aspartate and neuroprotective agents have been widely used for treating AD. However, these treatments can only modestly improve AD symptoms. The major challenge to finding effective treatments is the lack of complete understanding of the detailed mechanism underlying the pathogenesis of AD.

$\mathrm{A} \beta$ was recently recognized to play a major role in the pathogenesis of $\mathrm{AD}$. Furthermore, recent evidence indicated that tau hyperphosphorylation also plays an important role in AD pathology ( $\mathrm{Li}$ et al., 2007). In addition, other cleaved fragments of APP, such as the A $\beta$ precursor $\beta C T F$, were found to play a significant role in AD (Yang et al., 2003). Exploration of the relationship between tau phosphorylation and $\beta$ CTF processing might highlight the critical pathogenesis mechanism of AD. Therefore, the study of $\beta C T F$ processing in the hyperphosphorylated tau state is important to further explore the pathogenesis of $\mathrm{AD}$ and to develop new therapeutic targets. An in vitro study showed that $\beta C T F$ accumulated in the axons of neurons treated with OA, which suggested that the distribution of $\beta C T F$ was potentially involved in the pathogenesis of $\mathrm{AD}$ (Yoon et al., 2006). In our previous study, we found that the accumulation of $\beta C T F$ in a cell model of hyperphosphorylated tau led to cellular degeneration (Yu et al., 2008). However, little is known about the role of $\beta \mathrm{CTF}$ in vivo, which might exist in a more complicated state than it does in vitro. In this study, we investigated $\beta$ CTF processing in a rat model of tau hyperphosphorylation, and examined the effect of BACE1 inhibitor on $\beta C T F$ processing in vivo.

In the present study, we established an animal model of the tau hyperphosphorylation state by OA bilateral icv injection. OA is a selective and effective inhibitor of serine/threonine phosphatases 2A (Ishihara et al., 1989), and PP2A is the major tau phosphatase in the brain (Veeranna Shetty et al., 2000). By inhibiting PP2A, OA could induce an Alzheimer's-like tau hyperphosphorylation state in vitro and in brain slices of humans or rats (He et al., 2001; Alvarez-de-la-Rosa et al., 2005; Zhang and Simpkins, 2010). In vivo, OA could also cause tau hyperphosphorylation and neuronal cell death by both intraventricular and hippocampus microinfusion in rats (He et al., 2001). In our study, we found that rats administered with bilateral OA $i c v$ injection showed apparent tau phosphorylation and memory defects.

$\beta C T F$ is the cleaved fragment of APP that is processed by BACE1, and was found to accumulate in cell models of AD and in the AD brain (Jin et al., 2004). Therefore, $\beta C T F$ might be involved in the neuronal degeneration associated with $A D$, and the role of $\beta C T F$ in AD-related neurodegeneration has been studied previously (Lee et al., 2000). Fukuchi et al. (1993) demonstrated that expression of full-length cDNA of human $\beta C T F$ was toxic in cultured neuronal and non-neuronal cell lines. In addition, McPhie et al. (2001) showed that the apoptosis of neurons caused by mutations in the APP gene was mediated by $\beta$ CTF more than by $\mathrm{A} \beta$ in early onset $\mathrm{AD}$. $\beta \mathrm{CTF}$ exposure could induce an inflammatory reaction by the activation of mitogen-activated protein kinase pathways as well as by the transcription factor nuclear factor-kappa B (Bach et al., 2001). Moreover, $\beta$ CTF could bind to the Fe65 adapter protein and move to the nucleus, by which $\beta$ CTF might contribute to the pathogenesis of AD (Cao and Südhof, 2001). In the present study, Western blot analysis showed that $\beta C T F$ increased in the hippocampus of OA-treated rats, and these rats also showed memory defects. These results suggested that tau hyperphosphorylation contributed to $\beta C T F$ generation, which might have in turn contributed to memory defects in rats with hyperphosphorylated tau. 
Furthermore, we also aimed to determine the cause of the increase in $\beta \mathrm{CTF}$ in the hyperphosphorylated tau state and the effect of BACE1 inhibitor on $\beta C T F$ processing. We detected the expression of APP as well as the activity of the BACE1 in vivo. Accordingly, we found that the increase in $\beta C T F$ was mainly due to the overexpression of BACE1, and was not significantly associated with the overexpression of APP. In addition, we found that BACE1 inhibitor markedly reduced the production of total CTF (data not shown), suggesting that increased CTF from APP cleavage in the hyperphosphorylated tau state would be primarily composed of $\beta C T F$ rather than $\alpha \mathrm{CTF}$. In general, these results suggested that APP was cleaved by BACE1 rather than by $\alpha$-secretase in the state of tau hyperphosphorylation, and thus more $\beta C T F$ was generated. The hyperphosphorylated tau state resulted in a shift of the APP processing pathway; however, the level of APP did not change in vivo. It is interesting to note that in our previous in vitro study, treatment with BACE1 inhibitor led to an increase in APP in OA-treated neurons, whereas in vivo, we here found that APP did not increase in the hippocampal neurons of rats given BACE1 inhibitor. This discrepancy might be partially due to the difference between cells and tissues; however, the precise mechanism will be explored further.

The $\beta C T F$ antibody was found stained mainly in the nucleus, plaques, and neurites in neurons of AD patients' autopsied brains (Lahiri et al., 2002). In the cell model of hyperphosphorylated tau, $\beta \mathrm{CTF}$ mainly accumulated in axons, suggesting that the distribution of $\beta C T F$ in axons might be involved in the pathogenesis of AD. However, little was known about the impact of the $\beta \mathrm{CTF}$ distribution in the more complicated in vivo state. For this purpose, we analyzed its distribution with immunohistochemistry and found that $\beta$ CTF had accumulated in the axons of neurons and that it might be related to memory defects. We further showed that BACE1 inhibitor could reduce the deposition of $\beta C T F$ in axons and improve memory defects. In normal neurons, APP, secretases, and its cleaved fragments are delivered to nerve terminals by fast axonal transport along microtubules (Lazarov et al., 2005). Among all the secretase components analyzed in the axon, only $\alpha$-secretase was detected in APP-containing vesicles (Szodorai et al., 2009); however, APP and BACE1 were sorted into different carriers, and their vesicles differed in speed, direction of movement, and flux rate through axons (Goldsbury et al., 2006). This indicated that APP was cleaved by $\alpha$-secretase and that small amounts of $\alpha \mathrm{CTF}$ (C83) might be generated when APP is transported along the axon in normal neurons. Hyperphosphorylated tau was shown to hamper such axonal transport by microtubule disruption (Vershinin et al., 2007; Lapointe et al., 2009). It was presumed that the accumulation of hyperphosphorylated tau in the axons inhibited the transport of APP and secretases, which increased the chance of vesicles containing APP and BACE1, resulting in a shift in the processing of APP from the $\alpha$-secretase to the $\beta$-secretase pathway, consequently generating more neurotoxic $\beta C T F$, which becomes principally distributed in the axons. Further research is required to understand this process at the organelle level.

Our results showed that OA treatment led to an increase in $\beta \mathrm{CTF}$ as well as learning and memory defects in rats, which suggested that $\beta \mathrm{CTF}$ accumulation causes learning and memory deficits (Lee et al., 2006). We also found that BACE1 inhibitor ameliorated the memory defects resulting from changes in the quantity and distribution of neurotoxic $\beta C T F$ (Kimura et al., 2010). In-depth understanding of the involvement of $\beta C T F$ in neurodegeneration is an important determinant for the use of BACE1 inhibitor and $\gamma$-secretase inhibition as major therapeutic strategies for $\mathrm{AD}$; however, there is controversy about which one would be more appropriate (Golde and Younkin, 2001). Considering the effect of $\beta C T F$ on memory, the 
application of $\gamma$-secretase inhibitor should be used with caution; although it can decrease $\mathrm{A} \beta$, it might simultaneously increase the generation of $\beta C T F$ (Jiang et al., 2010).

In summary, tau hyperphosphorylation led to a shift in APP processing from the $\alpha$-secretase pathway to the $\beta$-secretase pathway. The levels of neurotoxic $\beta C T F$ increased and accumulated in axons, which could possibly facilitate the pathogenesis of AD. BACE1 inhibitor could decrease the overproduction and axon deposition of neurotoxic $\beta C T F$, thereby improving memory defects, which might be an effective target of AD. Together, these results suggest that OA-induced neurodegeneration merits further exploration to contribute to understanding of the pathogenesis of AD.

\section{ACKNOWLEDGMENTS}

Research supported by the Second Affliated Hospital of Harbin Medical University Doctors' Funds (\#BS2009-28).

\section{REFERENCES}

Alvarez-de-la-Rosa M, Silva I, Nilsen J, Perez MM, et al. (2005). Estradiol prevents neural tau hyperphosphorylation characteristic of Alzheimer's disease. Ann. N. Y. Acad. Sci. 1052: 210-224.

Bach JH, Chae HS, Rah JC, Lee MW, et al. (2001). C-terminal fragment of amyloid precursor protein induces astrocytosis. J. Neurochem. 78: 109-120.

Cao X and Südhof TC (2001). A transcriptionally [correction of transcriptively] active complex of APP with Fe65 and histone acetyltransferase Tip60. Science 293: 115-120.

Chang KA and Suh YH (2005). Pathophysiological roles of amyloidogenic carboxy-terminal fragments of the betaamyloid precursor protein in Alzheimer's disease. J. Pharmacol. Sci. 97: 461-471.

Choi SH, Park CH, Koo JW, Seo JH, et al. (2001). Memory impairment and cholinergic dysfunction by centrally administered $A \beta$ and carboxyl-terminal fragment of Alzheimer's APP in mice. FASEB J. 15: 1816-1818.

Fukuchi K, Sopher B, Furlong CE, Smith AC, et al. (1993). Selective neurotoxicity of COOH-terminal fragments of the beta-amyloid precursor protein. Neurosci. Lett. 154: 145-148.

Fukumoto H, Cheung BS, Hyman BT and Irizarry MC (2002). Beta-secretase protein and activity are increased in the neocortex in Alzheimer disease. Arch. Neurol. 59: 1381-1389.

Golde TE and Younkin SG (2001). Presenilins as therapeutic targets for the treatment of Alzheimer's disease. Trends Mol. Med. 7: 264-269.

Goldsbury C, Mocanu MM, Thies E, Kaether C, et al. (2006). Inhibition of APP trafficking by tau protein does not increase the generation of amyloid- $\beta$ peptides. Traffic 7: 873-888.

Gong CX and Iqbal K (2008). Hyperphosphorylation of microtubule-associated protein tau: a promising therapeutic target for Alzheimer disease. Curr. Med. Chem. 15: 2321-2328.

Hardy J (2006). Alzheimer's disease: the amyloid cascade hypothesis: an update and reappraisal. J. Alzheimers Dis. 9: 151-153.

He J, Yamada K, Zou LB and Nabeshima T (2001). Spatial memory deficit and neurodegeneration induced by the direct injection of okadaic acid into the hippocampus in rats. J. Neural Transm. 108: 1435-1443.

Ishihara H, Martin BL, Brautigan DL, Karaki H, et al. (1989). Calyculin A and okadaic acid: inhibitors of protein phosphatase activity. Biochem. Biophys. Res. Commun. 159: 871-877.

Jiang Y, Mullaney KA, Peterhoff CM, Che S, et al. (2010). Alzheimer's-related endosome dysfunction in Down syndrome is A $\beta$-independent but requires APP and is reversed by BACE-1 inhibition. Proc. Natl. Acad. Sci. U. S. A. 107: 1630-1635.

Jin LW, Shie FS, Maezawa I, Vincent I, et al. (2004). Intracellular accumulation of amyloidogenic fragments of amyloid- $\beta$ precursor protein in neurons with Niemann-Pick type $\mathrm{C}$ defects is associated with endosomal abnormalities. Am. J. Pathol. 164: 975-985.

Kimura R, Devi L and Ohno M (2010). Partial reduction of BACE1 improves synaptic plasticity, recent and remote memories in Alzheimer's disease transgenic mice. J. Neurochem. 113: 248-261. 
Lahiri DK, Kotwal GJ, Farlow MR, Sima A, et al. (2002). The role of the carboxyl-terminal fragments of amyloid precursor protein in Alzheimer's disease. Ann. N. Y. Acad. Sci. 973: 334-339.

Lapointe NE, Morfini G, Pigino G, Gaisina IN, et al. (2009). The amino terminus of tau inhibits kinesin-dependent axonal transport: implications for filament toxicity. J. Neurosci. Res. 87: 440-451.

Lazarov O, Morfini GA, Lee EB, Farah MH, et al. (2005). Axonal transport, amyloid precursor protein, kinesin-1, and the processing apparatus: revisited. J. Neurosci. 25: 2386-2395.

Lee JP, Chang KA, Kim HS, Kim SS, et al. (2000). APP carboxyl-terminal fragment without or with A $\beta$ domain equally induces cytotoxicity in differentiated PC12 cells and cortical neurons. J. Neurosci. Res. 60: 565-570.

Lee KW, Im JY, Song JS, Lee SH, et al. (2006). Progressive neuronal loss and behavioral impairments of transgenic C57BL/6 inbred mice expressing the carboxy terminus of amyloid precursor protein. Neurobiol. Dis. 22: 10-24.

Li JJ, Li ZY, Li HZ and Lin H (2007). Effect of hyperphosphorylated tau protein on the formation of brain amyloid. $J$. Clin. Neurol. 2: 119-121.

McPhie DL, Golde T, Eckman CB, Yager D, et al. (2001). $\beta$-Secretase cleavage of the amyloid precursor protein mediates neuronal apoptosis caused by familial Alzheimer's disease mutations. Brain Res. Mol. Brain Res. 97: 103-113.

Oddo S, Vasilevko V, Caccamo A, Kitazawa M, et al. (2006). Reduction of soluble Abeta and tau, but not soluble Abeta alone, ameliorates cognitive decline in transgenic mice with plaques and tangles. J. Biol. Chem. 281: 39413-39423.

Roberson ED, Scearce-Levie K, Palop JJ, Yan F, et al. (2007). Reducing endogenous tau ameliorates amyloid betainduced deficits in an Alzheimer's disease mouse model. Science 316: 750-754.

Simón AM, Schiapparelli L, Salazar-Colocho P, Cuadrado-Tejedor M, et al. (2009). Overexpression of wild-type human APP in mice causes cognitive deficits and pathological features unrelated to A $\beta$ levels. Neurobiol. Dis. 33: 369-378.

Szodorai A, Kuan YH, Hunzelmann S, Engel U, et al. (2009). APP anterograde transport requires Rab3A GTPase activity for assembly of the transport vesicle. J. Neurosci. 29: 14534-14544.

Veeranna Shetty KT, Takahashi M, Grant P and Pant HC (2000). Cdk5 and MAPK are associated with complexes of cytoskeletal proteins in rat brain. Brain Res. Mol. Brain Res. 76: 229-236.

Vershinin M, Carter BC, Razafsky DS, King SJ, et al. (2007). Multiple-motor based transport and its regulation by Tau. Proc. Natl. Acad. Sci. U. S. A. 104: 87-92.

Yang LB, Lindholm K, Yan R, Citron M, et al. (2003). Elevated $\beta$-secretase expression and enzymatic activity detected in sporadic Alzheimer disease. Nat. Med. 9: 3-4.

Yoon SY, Choi JE, Yoon JH, Huh JW, et al. (2006). BACE inhibitor reduces APP- $\beta$-C-terminal fragment accumulation in axonal swellings of okadaic acid-induced neurodegeneration. Neurobiol. Dis. 22: 435-444.

Yu CJ, Wang WZ and Liu W (2008). $\beta$-Secretase inhibitor increases amyloid- $\beta$ precursor protein level in rat brain cortical primary neurons induced by okadaic acid. Chin. Med.J. 121: 1439-1444.

Zhang Z and Simpkins JW (2010). Okadaic acid induces tau phosphorylation in SH-SY5Y cells in an estrogen-preventable manner. Brain Res. 1345: 176-181. 